Pacific Journal of Mathematics

BOUNDS IN THE NEWMAN PROBLEM FOR SECOND ORDER 


\title{
BOUNDS IN THE NEUMANN PROBLEM FOR SECOND ORDER UNIFORMLY ELLIPTIC OPERATORS
}

\author{
J. H. Bramble and L. E. Payne
}

1. Introduction. In this paper we derive certain a priori inequalities which are useful for obtaining bounds in the interior-Neumann problem for second order elliptic partial differential equations. In establishing these inequalities by our methods it is necessary to obtain lower bounds for the inverse of the Poincaré constant ( $\mu_{2}$ of eq. 3.3) and the first nonzero Steklov eigenvalue ( $p_{2}$ of eq. 3.11). An optimal Poincaré inequality for convex domains in $n$-dimensions was given by Payne and Weinberger [5], and a method for obtaining lower bounds for $p_{2}$ for $n$-dimensional star-shaped regions was indicated by Payne and Weinberger [3]. However, to the authors' knowledge, no explicit lower bounds for $p_{2}$ and $\mu_{2}$ for general $n$-dimensional regions have previously been given. Lower bounds for these constants which lead to the above mentioned inequalities in the Neumann problem are of interest in themselves and should prove useful in other applications.

For the special case of the Laplace equation other methods for deriving bounds for the Dirichlet integral in the Neumann problem appear in the literature (see [2], [6]). For starshaped regions a method similar to that proposed here was obtained in [3]. Bounds in exterior Neumann problems were given in [4].

2. Preliminary inequalities. Let $R$ be a simply connected bounded region with boundary $C$ in Euclidean $n$-space. In $R$ we assume that the operator $L$ given by

$$
L u \equiv\left(a^{i j} u_{, i}\right)_{, j}
$$

is a uniformly elliptic operator, defined for sufficiently smooth functions $u$. In (2.1), $i$ denotes partial differentiation with respect to the coordinate $x_{i}$ and the summation convention is assumed. The coefficient matrix $a^{i j}$ is symmetric and the condition of uniform ellipticity may be stated as follows: There exist positive constants $a_{0}$ and $a_{1}$ such that for every real vector $\left(\xi_{1}, \cdots, \xi_{n}\right)$, the relation

$$
a_{0} \sum_{i=1}^{n} \xi_{i} \xi_{i} \leqq a^{i j} \xi_{i} \xi_{j} \leqq a_{1} \sum_{i=1}^{n} \xi_{i} \xi_{i}
$$

is valid uniformly in $R$.

Received December 4, 1961. This research was supported in part by the United States Air Force through the Air Force Office of Scientific Research of the Air Resarch and Development Command under Contract No. AF 49 (638) 228. 
We consider now an arbitrary point of $R$ which we choose to be the origin. Let $S_{a}$ be the interior of a sphere of radius $a$, with center at the origin and such that $S_{a} \subset R$. The surface of the sphere will be called $\Sigma_{a}$. We denote by $R_{a}$ the region $R-\bar{S}_{a}$, where $\bar{S}_{a}$ is the closure of $S_{a}$.

Let $u$ be any sufficiently smooth function in $R+C$ and let $f^{i}$ be a sufficiently smooth vector field defined in $\bar{R}_{a}$. Then, by the divergence theorem, we have

$$
\oint_{o} f^{i} n_{i} u^{2} d s=-\oint_{\Sigma_{a}} f^{i} n_{i} u^{2} d s+\int_{R_{a}} f_{, i}^{i} u^{2} d v+2 \int_{R_{a}} f^{i} u u,{ }_{i} d v,
$$

where $n_{i}$ is the component of the unit normal directed outward on $C$. An application of the arithmetic-geometric mean inequality applied to the last term on the right of (2.3) yields

$$
\oint_{o} f^{i} n_{i} u^{2} d s \leqq-\oint_{\Sigma_{a}} f^{i} n_{i} u^{2} d s+\int_{R_{a}}\left(f_{{ }_{i}}^{i}+\frac{1}{\alpha} f^{i} f^{i}\right) u^{2} d v+\int_{R_{a}} \alpha u{ }_{, i} u_{, i} d v
$$

where $\alpha$ is some positive function in $R_{a}$.

We assume now that $f^{i}$ and $\alpha$ have been chosen so that

$$
\begin{aligned}
t \equiv f^{i} n_{i} & \geqq K_{1}>0 \text { on } C \\
-f^{i} n_{i} & \leqq K_{2} \text { on } \Sigma_{a} \\
f_{, i}^{i}+\frac{1}{\alpha} f^{i} f^{i} & \leqq 0 \text { in } R_{a},
\end{aligned}
$$

where $K_{1}$ and $K_{2}$ are constants. (We shall in a subsequent section construct vectors $f^{i}$ satisfying (2.5) for certain domains.) Using conditions (2.5) with (2.4) we have that

$$
\oint_{\sigma} t u^{2} d s \leqq K_{2} \oint_{\Sigma_{a}} u^{2} d s+\bar{\alpha} \int_{R_{a}} u_{, i} u_{, i} d v,
$$

where $\bar{\alpha}$ is an upper bound for $\alpha$ in $R_{a}$. Suppose that $u$ is normalized such that

$$
\oint_{\Sigma_{a}} u d s=0
$$

Then

$$
\oint_{\Sigma_{a}} u^{2} d s \leqq p_{2} \int_{S_{a}} u_{, i} u, i d v
$$

where $p_{2}$ is the first nonzero eigenvalue in the Steklov problem for the sphere $S_{a}$. That is 


$$
p_{2}=\min \frac{\int_{s_{a}} \chi_{, i} \chi_{, i} d v}{\oint_{\Sigma_{a}} \chi^{2} d s}
$$

where the minimum is taken over all sufficiently smooth functions in $S_{a}$ which satisfy (2.7). For the sphere of radius $a, p_{2}$ is explicitly given by

$$
p_{2}=1 / a .
$$

Combining (2.6) and (2.8) it follows that

$$
\oint_{o} t u^{2} d s \leqq K_{3} D(u, u) \equiv K_{3} \int_{R} u_{. i} u_{, i} d v,
$$

where $K_{3}=\max \left(a K_{2}, \bar{\alpha}\right)$, or using (2.5)

$$
\oint_{\sigma} u^{2} d s \leqq\left(K_{3} / K_{1}\right) D(u, u) .
$$

Now from the divergence theorem

$$
\oint_{o} x^{i} n_{i} u^{2} d s=n \int_{R} u^{2} d v+2 \int_{R} x^{i} u u_{, i} d v .
$$

Using the arithmetric-geometric mean inequality it follows easily that

$$
\int_{R} u^{2} d v \leqq \frac{2 r_{M}}{n} \oint_{o} u^{2} d s+\frac{4 r_{M}^{2}}{n^{2}} D(u, u),
$$

where $r_{M}$ is the maximum distance from the origin to $C$. Inequality (2.12) with (2.14) yields

$$
\int_{R} u^{2} d v \leqq K_{4} D(u, u) ，
$$

where

$$
K_{4}=\frac{2 r_{M}}{n}\left[K_{3} / K_{1}+\frac{2 r_{M}}{n}\right] .
$$

The preceding inequalities depended entirely on the existence of a vector field $f^{i}$ satisfying (2.5). In certain cases, as will be shown in a subsequent section, such a vector field can be explicitly constructed so as to yield explicit, easily computable constants $K_{1}, K_{2}$ and $K_{3}$.

In some cases it may be that for the region $R$ the vector field $f^{i}$ is quite difficult to construct. We can make use of an additional inequality to reduce the problem to that of obtaining an inequality of the form (2.12) for a subregion of $R$.

Let us divide the region $R$ into two disjoint subregions $R_{1}$ and $R_{2}$. 
These regions are to be separated by a surface $C^{\prime}$. The portion $C$ which is part of the boundary of $R_{i}$ will be denoted by $C_{i}, i=1,2$. Thus the boundary of $R_{i}$ will be $C_{i}+C^{\prime}$. We further assume that the subdivision has been made in such a way that $C_{1}$ is star shaped with respect to some point $P$ not in $R_{1}+C^{\prime}$. We choose $P$ to be the origin and apply the divergence theorem is $R_{1}$ to obtain

$$
\int_{\sigma_{1}+\sigma^{\prime}} x^{i} r^{-(n+1)} n_{i} u^{2} d s=-\int_{R_{1}} r^{-(n+1)} u^{2} d v+2 \int_{R_{1}} x^{i} r^{-(n+1)} u u_{, i} d v
$$

for any function $u$ sufficiently smooth in $R$. Defining $\hat{i}=x_{i} n_{i} / r$ and using the arithmetic-geometric mean inequality we obtain

$$
\oint_{\sigma_{1}} u^{2} d s \leqq \frac{1}{\hat{t}_{m}}\left(\frac{r_{M}}{r_{m}}\right)^{n} \oint_{\sigma^{\prime}} u^{2} d s+\frac{r_{m}}{\hat{t}_{m}}\left(\frac{r_{M}}{r_{m}}\right)^{n} D_{1}(u, u) .
$$

In (2.17) $r_{M}$ and $r_{m}$ denote upper and lower bounds for $r$ in $\bar{R}_{1}$ and $\hat{t}_{m}$ a lower bound for $\hat{t}$ on $C_{1} \cdot D_{1}(u, u)$ denotes the Dirichlet integral over $R_{1}$.

Now suppose that for $R_{2}$ we could find a vector field $f^{i}$ satisfying (2.5) relative to $R_{2}$ and obtain the inequality

$$
\oint_{C_{2}+C^{\prime}} u^{2} d s \leqq K_{3} / K_{1} D_{2}(u, u) .
$$

Then clearly (2.17), together with (2.18) would yield

$$
\oint_{o} u^{2} d s \leqq K_{5} D(u, u)
$$

where of course $u$ is assumed normalized with respect to $S_{a}$ in $R_{2}$, and $K_{5}$ is a constant.

It is now obvious that such a procedure could be repeated a finite number of times, finally reducing the region to one for which the inequality (2.12) may be more easily obtained. In particular if we iterate this procedure until the $q$ th region $R_{q}$ is star shaped, then, as we shall see in $\S 4$, a vector field $f^{i}$ for $R_{q}$ is easily constructed.

3. Lower bounds for eigenvalues. The first nonzero eigenvalue $\mu_{2}$ in the free membrane problem for $R$ satisfies

$$
\Delta \widetilde{v}+\mu_{2} \widetilde{v}=0 \text { in } R
$$

and

$$
\frac{\partial \widetilde{v}}{\partial n}=0 \text { on } C,
$$

where $\tilde{v}$ is the corresponding eigenfunction. It is well known that $\mu_{2}$ 
may be characterized by the minimum principle

$$
\mu_{2}=\min \frac{D(\varphi, \varphi)}{\int_{R} \varphi^{2} d v}
$$

for sufficiently smooth functions $\varphi$ satisfying

$$
\int_{R} \varphi d v=0
$$

and that $\widetilde{v}$ is the minimizing function. That is

$$
\mu_{2}=\frac{D(\widetilde{v}, \widetilde{v})}{\int_{R} \widetilde{v}^{2} d v}
$$

Now let $u=\widetilde{v}+c_{1}$ where

$$
c_{1}=-\frac{1}{\omega_{n} a^{n-1}} \int_{\Sigma_{a}} v d s
$$

$\omega_{n}$ denoting the surface area of the $n$-dimensional unit sphere. Then $u$ satisfies

$$
\int_{\Sigma_{a}} u d s=0
$$

and hence by (2.15)

$$
\int_{R} u^{2} d v \leqq K_{4} D(u, u)=K_{4} D(\widetilde{v}, \widetilde{v}) .
$$

But

$$
\int_{R} u^{2} d v=\int_{R} \widetilde{v}^{2} d v+c_{1}^{2} \int_{R} d v \geqq \int_{R} \widetilde{v}^{2} d v
$$

Thus

$$
\frac{1}{K_{4}} \leqq \frac{D(\widetilde{v}, \widetilde{v})}{\int_{R} \widetilde{v}^{2} d v}=\mu_{2}
$$

or $1 / K_{4}$ is a lower bound for $\mu_{2}$.

A lower bound is also easily obtained for $p_{2}$, the first nonzero eigenvalue in the Steklov problem for $R$. Let $w$ be the corresponding eigenfunction. Then we have that

$$
p_{2}=\frac{D(w, w)}{\oint_{\sigma} w^{2} d s}
$$


and

$$
\oint_{o} w d s=0
$$

Now let $u=w+c_{2}$ where

$$
c_{2}=-\frac{1}{\omega_{n} a^{n-1}} \oint_{\Sigma_{a}} w d s .
$$

Then

$$
\oint_{\Sigma_{a}} u d s=0
$$

and we may apply (2.12) to $u$. But by (3.14) we have that

$$
\oint_{o} u^{2} d s=\oint_{\sigma} w^{2} d s+c_{2}^{2} \oint_{\sigma} d s \geqq \oint_{\sigma} w^{2} d s .
$$

Thus from (2.12) and (3.15)

$$
K_{1} / K_{3} \leqq \frac{D(w, w)}{\oint_{o} w^{2} d s}=p_{2},
$$

which gives the desired lower bound for $p_{2}$.

4. Bounds in the Neumann problem for $L$. We assume now that $\psi$ is any sufficiently smooth function in $R+C$. We shall obtain bounds for the generalized Dirichlet integral, $A(\psi, \psi)$, given by

$$
A(\psi, \psi)=\int_{R} a^{i j} \psi_{, i} \psi,{ }_{, j} d v
$$

in terms of $L \psi$ in $R$ and

$$
\frac{\partial \psi}{\partial \nu} \equiv a^{i j} n_{i} \psi_{, j} \text { on } C .
$$

We take $u=\psi+c_{3}$ where

$$
c_{3}=-\frac{1}{\omega_{n} a^{n-1}} \oint_{\Sigma_{a}} \psi d s .
$$

As before

$$
\oint_{\Sigma_{a}} u d s=0
$$

Now by Green's identity 


$$
A(u, u)=\oint_{o} u \frac{\partial \psi}{\partial \nu} d s-\int_{R} u L \psi d v
$$

We have used the fact that $u$ and $\psi$ differ only by a constant. By Schwarz's inequality we have that

$$
\begin{aligned}
A(u, u) \leqq & \left(\oint_{\sigma} t u^{2} d s\right)^{1 / 2}\left(\oint_{\sigma} t^{-1}\left(\frac{\partial \psi}{\partial \nu}\right)^{2} d s\right)^{1 / 2} \\
& +\left(\int_{R} u^{2} d v\right)^{1 / 2}\left(\int_{R}(L \psi)^{2} d \nu\right)^{1 / 2} .
\end{aligned}
$$

Because of (4.4) inequalities (2.11) and (2.15) are applicable and we obtain that

$$
A(\psi, \psi)^{1 / 2} \leqq\left(\frac{K_{3}}{a_{0}}\right)^{1 / 2}\left(\oint_{o} t^{-1}\left(\frac{\partial \psi}{\partial \nu}\right)^{2} d s\right)^{1 / 2}+\left(\frac{K_{4}}{a_{0}}\right)^{1 / 2}\left(\int_{R}(L \psi)^{2} d v\right)^{1 / 2}
$$

since

$$
D(u, u) \leqq \frac{1}{a_{0}} A(u, u)=\frac{1}{a_{0}} A(\psi, \psi)
$$

The inequalities of this section and $\S 2$ together with a mean value inequality given in [1] give immediately interior pointwise bounds for $\psi+c_{3}$.

As an application of the results of this section we note here that (4.7) may be used in conjunction with the Rayleigh-Ritz procedure to yield close bounds for the Dirichlet integral in a specific Neumann problem c.f. [1].

5. Construction of the vector field. We shall show in some cases how to construct a vector field satisfying (2.5).

(a) Star shaped regions.

We consider the case where $C$ is star shaped with respect to some point. We choose this point to be the origin. Then if we take

$$
f^{i}=x^{i} r^{-(n+1)}
$$

and

$$
\alpha=r^{-(n-1)}
$$

We have that

$$
t=f^{i} n_{i}=x^{i} n_{i} r^{-(n+1)} \geqq h_{m} r_{M}^{-(n+1)} \text { on } C
$$

where $h(P)$ is the distance from the origin to the tangent plane at a point $P$ on $C$ and $h_{m}$ is the minimum of this function. The condition 
of star-shapedness insures that $h_{m}>0$. Since $n_{i}=-x^{i} / a$ on $\Sigma_{a}$ it follows that

$$
-f^{i} n_{i}=a^{-n} \text { on } \Sigma_{a}
$$

and

$$
f_{, i}^{i}+\frac{1}{\alpha} f^{i} f^{i}=0 \text { in } R_{a}
$$

In this case, taking $a=r_{m}$, we obtain

$$
\mu_{2} \geqq \frac{n}{2 r_{M}^{2}\left[\left(\frac{r_{M}}{r_{m}}\right)^{n-1} \frac{r_{M}}{h_{m}}+\frac{2}{n}\right]}
$$

and

$$
p_{2} \geqq \frac{1}{r_{M}}\left[\left(\frac{r_{m}}{r_{M}}\right)^{n-1} \frac{h_{m}}{r_{M}}\right] .
$$

A different method for obtaining a lower bound for $p_{2}$ for star shaped regions has been indicated by Payne and Weinberger [3]. For convex region Payne and Weinberger [5] also obtained the optimal lower bound $\mu_{2} \geqq \pi^{2} d^{-2}$ where $d$ is the diameter of $R$.

(b) Smooth boundaries.

Let $R$ be a region whose boundary $C$ has continuous curvature. Call the largest principal curvature at a point $P$ of $C, K_{x}(p)$. Let $\rho(p)$ be the radius of a sphere which is tangent to $C$ at $P$ and contained in $R$. In addition we require $\rho(p)$ to be less than $K_{M}(p)^{-1}$. Denote by $\bar{K}$ a bound for the maximum of $\rho(p)^{-1}$ for $P \in C$. We consider the family of parallel surfaces

$$
N(x)=N\left(x^{1}, \cdots, x^{n}\right)=\text { constant }
$$

with $C$ given by

$$
N(x)=0
$$

and

$$
0 \leqq N(x) \leqq 1 / \bar{K}
$$

The outward normal vector $n_{i}$ is defined in this strip and satisfies

$$
n_{i, i}(x)=J(x)
$$

where $J(x)$ is the average curvature of the surface given by $N(x)$ at the point $x=\left(x^{1}, \cdots, x^{n}\right)$ c.f. $[7$, p. 3]. We assume also that $\bar{K}$ is chosen so that 


$$
J(x) \leqq \bar{K}
$$

The above conditions and definitions involve the smoothness of $C$ and essentially the thickness of $R$. We impose a further condition on the shape of $R$.

We assume that there is a point, which we choose to be the origin, such that

$$
\frac{x^{i} n_{i}}{r} \geqq-p+\beta>-p>-1
$$

for some constants $p$ and $\beta>0$ in the strip $0 \leqq N(x) \leqq 1 / \bar{K}$. In this case $f^{i}$ may be chosen as

$$
f^{i}=\left\{\begin{array}{l}
{\left[p n_{i}(1-\bar{K} N(x))+x^{i} / r\right] r^{-q}, 0 \leqq N(x) \leqq 1 / \bar{K}} \\
\frac{x^{i}}{r} r^{-q}, \text { otherwise }
\end{array}\right.
$$

with $q$ to be determined. Condition (5.12) means that there is an open subset $\Omega$ of $R$ which has the property that no ray from the boundary in the direction of the outward normal intersects $\Omega$.

Let a now be chosen so that $S_{a}$ does not intersect the boundary strip.

Now on $C$

$$
f^{i} n_{i}=\left[p+\frac{x^{i} n_{i}}{r}\right] r^{-q} \geqq \beta r_{M}^{-q} .
$$

For $0 \leqq N(x) \leqq 1 / \bar{K}$

$$
\begin{aligned}
f_{, i}^{i}=\{p[J(1-\bar{K} N(x))+\bar{K}] r & +n-1-q \\
& {\left.\left[p \frac{x_{i} n_{i}}{r}(1-\bar{K} N(x))+1\right]\right\} r^{-(q+1)}, }
\end{aligned}
$$

since $n_{i}\left(\partial / \partial x_{i}\right) N=-1$. Because of (5.12) and the fact that $0 \leqq 1-$ $\bar{K} N(x) \leqq 1$ we have that

$$
f_{, i}^{i} \leqq\left\{2 \bar{K} r_{M}+n-1-q\left(1-p^{2}\right)\right\} r^{-(q+1)} .
$$

Now if we choose

$$
q=\frac{2 \bar{K} r_{M}+n+3}{1-p^{2}}
$$

it follows that

$$
f_{, i}^{i} \leqq-4 r^{-(q+1)} \leqq-4 r_{M}^{-(q+1)}
$$

in the boundary strip. In the remaining part of $R_{a}$ we have, since 
$q \geqq n+3$

$$
f_{, i}^{i}=[n-(q+1)] r^{-(q+1)} \leqq-4 r_{M}^{-(q+1)} .
$$

Now choose $\alpha=r^{1-q}$. Then

$$
f_{, i}^{i}+\frac{1}{\alpha} f^{i} f^{i} \leqq 0 \text { in } R_{a} .
$$

On $\Sigma_{a}$

$$
-f^{i} n_{i}=a^{-q}
$$

In this case we have

$$
\mu_{2} \geqq \frac{n}{2 r_{M}^{2}\left[\frac{1}{\beta}\left(\frac{r_{M}}{a}\right)^{q-1}+\frac{2}{n}\right]}
$$

and

$$
p_{2} \geqq \beta\left(\frac{a}{r_{M}}\right)^{q-1} 1 / r_{M}
$$

(c) Boundaries with star-shaped irregularities.

Suppose now that the boundary $C$ consists of two parts $C_{1}$ and $C_{2}$ where $C_{1}$ is smooth and $C_{2}$ is star-shaped with respect to the chosen origin. We assume that the closure of the interior of $C_{2}$ contains $C_{2}$. For example, in two dimensions, the components of $C_{2}$ cannot be isolated points. Let $\bar{K}$ now be defined relative to $C_{1}$. Denote by $R_{1}$ the region consisting of $R$ minus the strip adjacent to $C_{1}$. We suppose that $\bar{K}$ is large enough (the strip small enough) to make $R_{1}$ connected.

We assume that $\beta$ is such that on $C_{2}$

$$
\frac{x^{i} n_{i}}{r} \geqq \beta \text {. }
$$

Then in place of $(5.13)$ we have

$$
f^{i}= \begin{cases}{\left[p n_{i}(1-\bar{K} N(x))+\frac{x^{i}}{r}\right] r^{-q},} & \text { in } R-R_{1} \\ \frac{x^{i}}{r} r^{-q}, & \text { in } R_{1} .\end{cases}
$$

Since in the identity (2.3) it is only necessary that $f^{i}$ have a continuous normal component on the boundaries of subregions of $R_{a}$ this definition of $f^{i}$ has sufficient smoothness properties.

In this case we again have the inequalities (5.22) and (5.23). 


\section{BIBLIOGRAPHY}

1. J. H. Bramble and L. E. Payne, Bounds for solutions of second order elliptic partial differential equations, Contributions to Diff. Eqtns. (to appear).

2. J. B. Diaz and A. Weinstein, Schwarz' inequality and the methods of Rayleigh-Ritz and Trefftz, J. Math. and Physics, 26 (1947), 133-136: see also J. B., Diaz, Upper and lower bounds for quadratic integrals, Collectanea Mathematica, 4 (1951), 3-50.

3. L. E. Payne and H. F. Weinberger, New bounds in harmonic and biharmonic problems, J. Math. Phys. (1955), 291-307.

4. - New bounds for solutions of second order elliptic partial differential equations, Pacific. J. Math., 8 (1958), 551-573.

5. — - An optimal Poincaré inequality for convex domains, Arch. Rat. Mech. Analy., 5 (1960), 280-292.

6. J. L. Synge, The method of hypercircle in function-space for boundary value problems, Proc. Roy. Soc. London, A 191 (1947), 447-467; se also J. L. Synge, The hypercircle in mathematical physics, Cambridge Univ. Press (1957).

7. C. E. Weatherburn, Differential geometry in three dimensions, Cambridge Univ. Press, vol. II (1930).

INSTITUTE FOR FLUID DyNAMiCs AND APPLIED MATHEMATICS

UNIVERSITY OF MARYLAND

College Park, Maryland 



\title{
PACIFIC JOURNAL OF MATHEMATICS
}

\author{
EDITORS
}

RalPh S. Phillips

Stanford University

Stanford, California

M. G. Arsove

University of Washington

Seattle 5 , Washington
A. L. Whiteman

University of Southern California Los Angeles 7, California

LOWell J. PAIGE

Unıversity of California

Los Angeles 24, California

\section{ASSOCIATE EDITORS}
E. F. BECKENBACH
D. DERRY
H. L. ROYDEN
E. G. STRAUS
T. M. CHERRY
M. OHTSUKA
E. SPANIER
F. WOLF

\section{SUPPORTING INSTITUTIONS}

\author{
UNIVERSITY OF BRITISH COLUMBIA \\ CALIFORNIA INSTITUTE OF TECHNOLOGY \\ UNIVERSITY OF CALIFORNIA \\ MONTANA STATE UNIVERSITY \\ UNIVERSITY OF NEVADA \\ NEW MEXICO STATE UNIVERSITY \\ OREGON STATE UNIVERSITY \\ UNIVERSITY OF OREGON \\ OSAKA UNIVERSITY \\ UNIVERSITY OF SOUTHERN CALIFORNIA
}

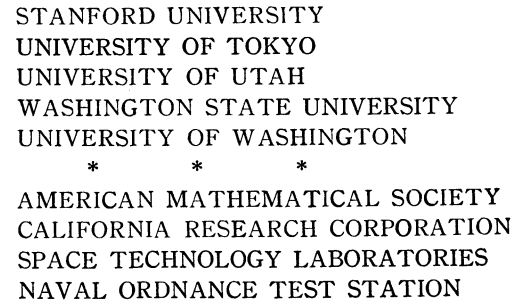

Mathematical papers intended for publication in the Pacific Journal of Mathematıcs should be typewritten (double spaced), and the author should keep a complete copy. Manuscripts may be sent to any one of the four editors. All other communications to the editors should be addressed to the managing editor, L. J. Paige at the University of California, Los Angeles 24, California.

50 reprints per author of each article are furnıshed free of charge; additional copies may be obtained at cost in multiples of 50 .

The Pacific Journal of Mathematics is published quarterly, in March, June, September, and December. Effective with Volume 13 the price per volume (4 numbers) is $\$ 18.00$; single issues, $\$ 5.00$. Special price for current issues to individual faculty members of supporting institutions and to individual members of the American Mathematical Society: $\$ 8.00$ per volume; single issues $\$ 2.50$. Back numbers are available.

Subscriptions, orders for back numbers, and changes of address should be sent to Pacific Journal of Mathematics, 103 Highland Boulevard, Berkeley 8, California.

Printed at Kokusai Bunken Insatsusha (International Academic Printing Co., Ltd.), No. 6 , 2-chome, Fujimi-cho, Chiyoda-ku, Tokyo, Japan.

PUBLISHED BY PACIFIC JOURNAL OF MATHEMATICS, A NON-PROFIT CORPORATION

The Supporting Institutions listed above contribute to the cost of publication of this Journal, but they are not owners or publishers and have no responsibility for its content or policies. 


\section{Pacific Journal of Mathematics}

\section{Vol. 12, No. $3 \quad$ March, 1962}

Alfred Aeppli, Some exact sequences in cohomology theory for Kähler

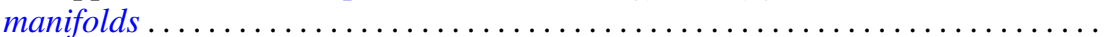

Paul Richard Beesack, On the Green's function of an N-point boundary value

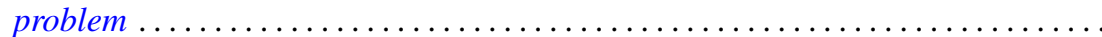

James Robert Boen, On p-automorphic p-groups....

James Robert Boen, Oscar S. Rothaus and John Griggs Thompson, Further results

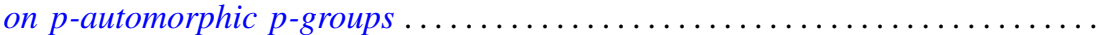

James Henry Bramble and Lawrence Edward Payne, Bounds in the Neumann problem for second order uniformly elliptic operators ..................

Chen Chung Chang and H. Jerome (Howard) Keisler, Applications of ultraproducts of pairs of cardinals to the theory of models .........................

Stephen Urban Chase, On direct sums and products of modules ................

Paul Civin, Annihilators in the second conjugate algebra of a group algebra .......

J. H. Curtiss, Polynomial interpolation in points equidistributed on the unit

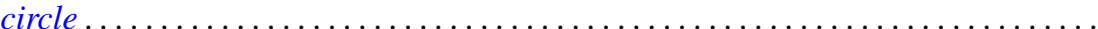

Marion K. Fort, Jr., Homogeneity of infinite products of manifolds with

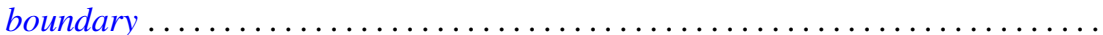

James G. Glimm, Families of induced representations . . . . . . . . . . . . . . .

Daniel E. Gorenstein, Reuben Sandler and William H. Mills, On almost-commuting

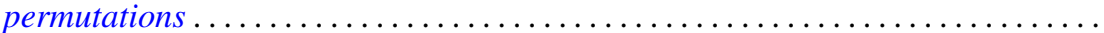

Vincent C. Harris and M. V. Subba Rao, Congruence properties of $\sigma_{r}(N) \ldots \ldots \ldots$

Harry Hochstadt, Fourier series with linearly dependent coefficients . . . . . . . . . . 925

Kenneth Myron Hoffman and John Wermer, A characterization of $C(X)$. .

Robert Weldon Hunt, The behavior of solutions of ordinary, self-adjoint differential equations of arbitrary even order...

Edward Takashi Kobayashi, A remark on the Nijenhuis tensor

David London, On the zeros of the solutions of $w^{\prime \prime}(z)+p(z) w(z)=0$

Gerald R. Mac Lane and Frank Beall Ryan, On the radial limits of Blaschke products...

T. M. MacRobert, Evaluation of an E-function when three of its upper parameters

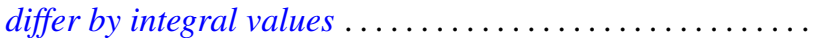

Robert W. McKelvey, The spectra of minimal self-adjoint extensions of a symmetric operator

Adegoke Olubummo, Operators of finite rank in a reflexive Banach space. .

David Alexander Pope, On the approximation of function spaces in the calculus of variations

Bernard W. Roos and Ward C. Sangren, Three spectral theorems for a pair of singular first-order differential equations...............

Arthur Argyle Sagle, Simple Malcev algebras over fields of characteristic zero .

S. C. Tang, Some theorems on the ratio of empirical distribution to the theoretical distribution

Robert Charles Thompson, Normal matrices and the normal basis in abelian number fields.

Howard Gregory Tucker, Absolute continuity of infinitely divisible distributions ... 